\title{
SIMULINK MODELLING AND CONTROLLER DESIGN FOR AN AUTO CRUISE
}

\section{R. SHASHI KUMAR REDDY ${ }^{1}$, M.S. TEJA ${ }^{2}$, RITESH KUMAR ${ }^{3} \&$ ADLARAJESH ${ }^{4}$}

${ }^{1}$ Assistant Professor and Head, Department of Electrical \& Electronics Engineering, Sumathi Reddy Institute of Technology for Women, Warangal, Telangana, India

${ }^{2}$ Assistant Professor, Department of Electrical \& Electronics Engineering, Sumathi Reddy Institute of Technology for Women, Warangal, Telangana, India

${ }^{3}$ Assistant Professor, Department of Electrical \& Electronics Engineering, SR Engineering College, Warangal, Telangana, India

${ }^{4}$ Assistant Professor, Department of Mechanical Engineering, Sumathi Reddy Institute of Technology for Women, Warangal, Telangana, India

\begin{abstract}
This paper presents the output characteristics of different control approaches that are developed in a cruise control system. It is broadly used because it is quite simple to understand and yet the control technique evolves classical and new design methods. The mathematical modeling for linear and nonlinear dynamics model of the auto cruise is developed in this paper. For linear model design, it requires proportional plus integral plus derivative controller, artificial intelligence controller and state space input and output equations. For nonlinear model with disturbance effect design, feed forward PID controller is developed in order to nullify the wind disturbance and gravitational effect. Finally, a comparison of the output of linear and nonlinear controller on the basis of obtained simulation results.

KEYWORDS: Fuzzy Logic Controller, Cruise Control System, PID with Feed Forward Controller, Linear and Nonlinear Systems, Simulink
\end{abstract}

Received: Jun 05, 2020; Accepted: Jun 25, 2020; Published: Jul 31, 2020; Paper Id.: IJMPERDJUN2020544

\section{INTRODUCTION}

Cruise is a control system with the controls in the motor vehicle of automatically over the throttle to the car to maintain a set by the driver constant. The purpose of the system is to maintain the desired speed accurately. A modern automation control loop takes over the control of the throttle, which is normally directed by the driver with the vehicle speed of the valve [I].

In the modern designs of the controlling applications of the turned on before use in some designs it is always enables as "ON" switch that must be pressed in the vehicle to be started. Many of the designs have to be in ON condition and must be set for the resume, accelerate and coast functions. Some may also have a cancel button. In the previous versions of designs a dial to set speed choice and the other way of control is by pressing in the brake will disable the system. Some driver can change the speed without any problem from the system. The features of the system built on steering is easy for driver to control the system.

The driver manually controls the cruise to normal speed and with the help of a button he can set the cruise to present speed. An auto cruise takes its speed signal from speedometer, rotating shaft and speed sensor of wheels from the engine's revolutions for minute. Cruise control maintain certain speed, otherwise systems do not use cruise. Vehicles maintain the speed with the help of an arrangement of throttle cable, solenoid, vaccum driven servo mechanism [II]. Auto cruise with memory feature to resume the set speed after using brakes. 
The latest models equipped with electronic sensors, throttle, cruise control can be integrated into the vehicle's engine management system. With the help of adaptive systems it can be reduces the speed automatically when there is a vehicle in front and it can adjust the speed limit decreases automatically [III]. It is the major advantage for drivers when they are in unknown areas.

\section{CRUISE CONTROL SYSTEM MODELLING}

From the model of a car, there are several important points that need to be discussed or analyzed in deriving a block diagram of the model. These points are actually the process of defining the input and output signals of a system, determination of the forces that act upon the system and the properties of the system itself that are significant enough in affecting the performance of the system [IV].

Firstly, the most important step in building a block diagram of the model is to define input and output signals. It is obvious now that the input signal of the model is desired speed that drivers intend to constantly drive the car. The output signal is the real speed at which the car is moving. The output speed will be the feedback to the input to be compared with the desired speed and the difference is calculated to be an error signal.

Secondly, disturbances causes by wind gust and the gravity acceleration must be included in the block diagram because they directly affect the speed of the car. These disturbances are actually opposing forces that reduced the forward force that accelerates the car [V]. Due to this reasoning, the forces should be injected into the feedback path. Hence, it is calculated into error signal together with the output signal.

Thirdly, in the original car model, the engine of the car produces the drive force that accelerates the car. The engine is responsible to carry out the task of increasing or decreasing the speed of the car. In other words, the performance of the car depends on the capability of the engine to provide the required drive force that moves the car at any desired speed. Therefore, the overall block diagram of the model needs to include the transfer function that represents the engine.

Fourthly, the net force of the car causes the car of certain mass to move with certain acceleration. Therefore, the output signal of the system is indeed in terms of force. The desired output signal of the system is speed but bit is not in the present case. To obtain speed, acceleration is needed because speed can be computed from the integral of the acceleration. Hence, the net must be divided by the mass of the car that acceleration can be the output of the system. In other words, the forward path of the block diagram should consist of constant with value equal to the reciprocal of the mass of the car. The constant is placed in front of the net force so that it will be immediately multiplied to give out acceleration [VI].

Finally, speed must be obtained from the acceleration of the car. Fortunately, since acceleration is the first derivative of the speed or similarly, speed is the first integral of the acceleration, an integrator should be introduced to provide an integral effect to the output path in order to transform the acceleration into speed. By placing the integral at the appropriate position in the block diagram [VII], the problem of obtaining speed from the acceleration is solved and the output is indeed the speed that is to be measured.

After defining all those variables, force and properties of the system the resulting block diagram demonstrated by figure (i). 


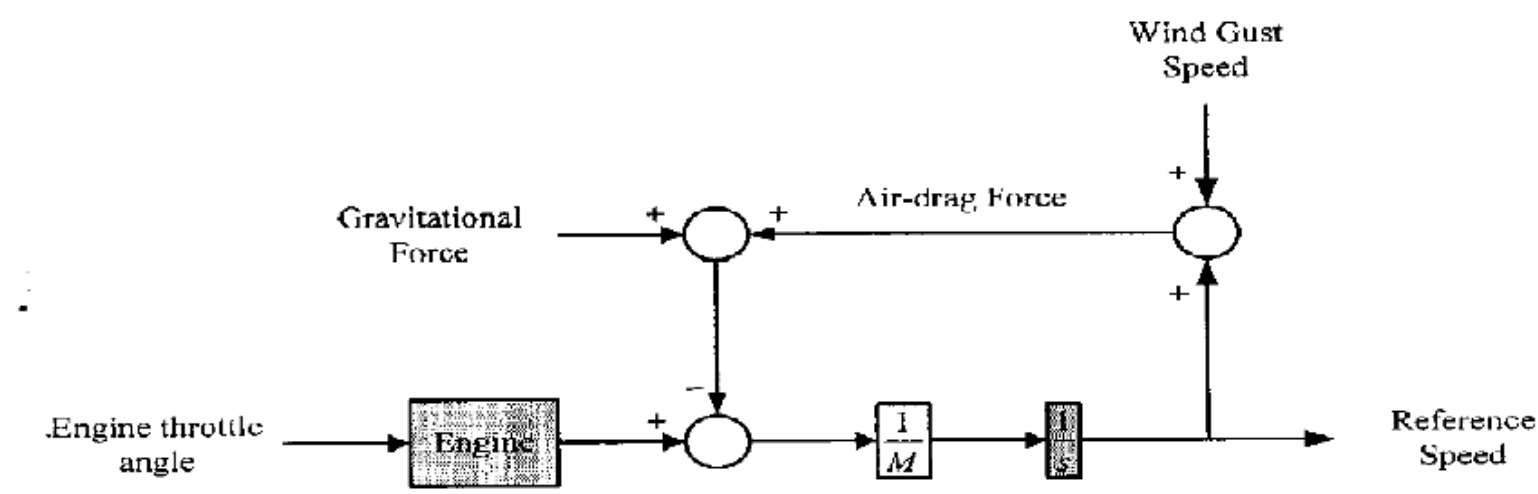

Figure (i): Schematic Diagram Illustrating All Variables

However, Lewis and Yang, gave a more detailed block diagram. The block diagram indicates precise components of the forces, the engine transfer function and the other important properties of the block diagram that was thoroughly discussed before. The formulae for both the air-drag force and gravitational force are given specifically. There is also a saturation limit for the drive force of the engine. The upper limit is given by $F_{d m a x}$ and the lower limit is given by $F_{d m i n}$. And integrator exists in the forward path of the block, providing the integral effect [VIII], to convert acceleration into speed. The wind gust speed is added to the feedback path together with the output speed, which is later computed to the air drag force. This air-drag force is then added up with the gravitational force to produce the opposing that decreases the speed of the car. The block diagram presented by Lewis and Yang is shown in figure (ii).

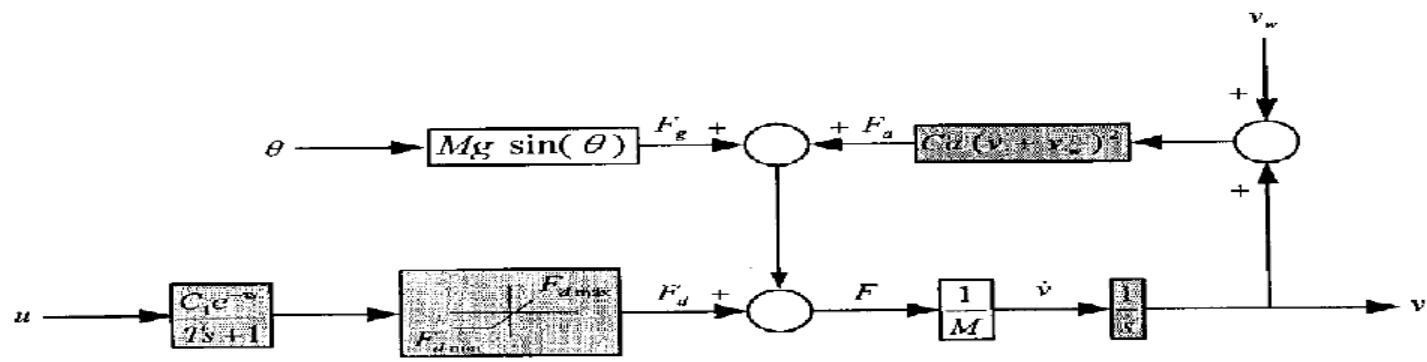

Figure (ii): Schematic Diagram of Lewis and Yang

From the block diagram the desired speed is represented by variable $u$ and the out represented by $v$. The wind gust is symbolized by variable $v_{w}$ and the grading is shown by the symbol $\theta$. The symbol $F, F_{d}, F_{g}$ and $F_{a}$ represent net force, the drive force, the gravitational force and the air-drag force respectively. Since this block diagram contains all the necessary information needed, as previously discussed in the process of deriving a general block diagram of the model [IX]. It will be used throughout the research.

\section{Values of Parameters and Constants}

Conveniently Lewis and Yang provided all of the values of constants and parameters used in the schematic block diagram. However, some values need to be modified so that the block diagram could represent the same model with slightly different values just to provide computing and calculating challenges rather that reusing the identical values. Table (i) gives the values of the constants of the parameters. 
Table (i)

\begin{tabular}{|c|c|}
\hline Symbols & Values \\
\hline $\mathrm{C}_{1}$ & 743 \\
\hline $\mathrm{C}_{\mathrm{a}}$ & $1.19 \mathrm{~N} /(\mathrm{m} / \mathrm{s})^{2}$ \\
\hline $\mathrm{F}_{\mathrm{dmax}}$ & $+3500 \mathrm{~N}$ \\
\hline $\mathrm{F}_{\mathrm{dmin}}$ & $-3500 \mathrm{~N}$ \\
\hline $\mathrm{G}$ & $9.81 \mathrm{~m} / \mathrm{s}^{2}$ \\
\hline $\mathrm{M}$ & $1500 \mathrm{~kg}$ \\
\hline $\mathrm{T}$ & $1 \mathrm{~s}$ \\
\hline
\end{tabular}

Notice that the values for $\theta$ and $v_{w}$ are not specified. This is because the values of the grading changes as the car moves $[\mathrm{X}]$. In other words, the grading is not similar for different hills. Similarly the wind gust may also change frequently.

\section{Transfer Function of the Model}

From the model of the system, a transfer function needs to be derived to represent the system mathematically. This mathematical expression will use extensively through this research for many purposes such as analyzing transient response, determining stability and controllability. The expression of the transfer function is given by equation as follows

$$
\frac{C^{*}(s)}{R(s)}-G(s)=\frac{b_{m} s^{m}+b_{m-1} s^{m-1}+\ldots b_{0}}{a_{n} s^{n}+a_{n-1} s^{n-1}+\ldots a_{0}}
$$

\section{Transfer Function through State Space Approach}

From the block diagram of figure (ii) the transfer function can be written through state space approach by selection of state variables as speed and force. Hence, Therefore,

$x_{l}=v$

$x_{2}=F_{d}$

\section{State and Output Equations}

It is very important to set all initial conditions to zero to develop the following state and output equations of the system.

$v=\left(F_{d}-C_{a} v^{2}\right) / M$

$F_{d}=\left(C_{l} u(t-T)-F_{d}\right)$

$y=v$

However, a problem of non linearity arises. There is a squared term in the equation. One way to linearize this problem is to linearize all of the state equations by differentiating both left and right hand sides of the equations with $M, \mathrm{C}_{\mathrm{a}}$, $\mathrm{C}_{1}$, T and $v$ remain constant $[\mathrm{XI}]$. After differentiating the state equations become

$$
\begin{aligned}
& \frac{d d}{d t} \dot{v}-\frac{1}{M}\left(-2 C_{d} v \bar{n} x+{ }_{d} F_{d}\right) \\
& \frac{d}{d l t} F_{i}-\frac{1}{T}\left(C_{1} u(t-T)-F_{d}\right)
\end{aligned}
$$


and the output equation becomes

$y=\delta v$

where,

$\delta v$ is the discrete output, $\delta F_{d}$ is the discrete force, $\delta u(t-T)$ is the time delay of the engine.

\section{The Transfer Function}

To find the TF, taking the ILT of the above equations yields.

$$
\begin{aligned}
& s \Delta V(s)-v(0)=\frac{1}{M}\left(-2 C_{d} v \Lambda V(s)+\Delta F_{d}(s)\right) \\
& s \Delta F_{d}(s)-F_{d}(0)=\frac{1}{T}\left(C_{1}\left(\Delta U(s) e^{-\pi}\right)-\Delta F_{d}(s)\right) \\
& Y(s)=\Lambda V(s)
\end{aligned}
$$

The rule of thumb in finding a transfer function is to set all the initial conditions to be zero. Applying this to the above equations yield

$$
\begin{aligned}
& s \wedge V(s)=\frac{1}{M}\left(-2 C_{a} \nu \Lambda V(s)+\Delta f_{d}^{\prime}(s)\right) \\
& s \Delta F_{d}(s)=\frac{1}{T}\left(C_{1}\left(\Delta U(s) e^{-\Phi}\right) \quad \Delta F_{d}(s)\right) \\
& \vdots(s)=\Delta V(s)
\end{aligned}
$$

Eliminating $\Delta \mathrm{F}_{\mathrm{d}}(\mathrm{s})$ by substitution yields the expression of the state-equation in terms of $\Delta \mathrm{V}(\mathrm{s})$ and $\Delta \mathrm{U}(\mathrm{s})$. Finally the $\mathrm{TF}$ is $\Delta \mathrm{V}(\mathrm{s}) / \Delta \mathrm{U}(\mathrm{s})$.

$$
\frac{\Delta V(s)}{\Delta U(s)}=\frac{C_{1} e^{-\pi}}{\left(s M+2 C_{n} v\right)(s T ! 1)}
$$

Rearranging the transfer function yields

$$
\frac{\Delta V(s)}{\Delta U(s)}=\frac{C_{1} e^{-z}}{\left.\left(s+\frac{2 C_{n} v}{M}\right)^{\prime} s+\frac{1}{T}\right)}
$$

This form of transfer function is clearly not linear since a factor of $e^{-t s}$ exists in the numerator. For design purpose, it is better if a transfer function is linear because it makes design processes much easier by reducing the complexity of calculation introduced by the non-linear elements in the transfer function [XII]. Thus, the above transfer function should be linearized for the purpose of design model.

\section{Linearization of Transfer Function}

A non-linear transfer function is complicated to analyze. Therefore, it is a common that non-linear transfer function is 
linearized before the system that is represented by the transfer can be analyzed. So far, the only-linear element in the transfer function of the system is the time delay. This time delay must be linearized to obtain a linear transfer function [XIX].

\section{Approximation of Time Delay}

Existence in time delay in any system leads to a much more complicated calculations and analysis compared to systems without delays. To avoid the problem, time delays need to be approximated to a rather much simple mathematical expression. There are many ways that can be use to approximate the time delays such as Pade approximation and Power Series Expansion approximation [XIII], Lewis and Yang used the power series expansion and approximation method. The derivation of the approximation is clearly shown by Bishop and Dorf. According to the power series expansion, the approximation of the time delays eventually is given by

$$
e^{-\tau} \approx \frac{1}{1+\tau s}
$$

Notice that it is actually being approximated to be a pole at $s=-1 / t$. Thus, this new pole will introduce a new effect to the transfer function. Particularly, the system order will be increased by one. Previously, the system was of a second-order but after applying the series expansion approximation, it becomes a third order system [XIV]. This change will somehow alter the characteristics of the system but it would not be significant if this new pole is at least five times farther than real part of the dominant second-order poles.

With the help of power series method, the time delay $[\mathrm{XV}]$ of the TF of the system is approximated to be

$$
e^{-s} \approx \frac{1}{1+\tau s}=\frac{\frac{1}{\tau}}{s+\frac{1}{\tau}}
$$

Substitute the above equation into the TF, we get

$$
\frac{\Delta V(s)}{\Delta U(s)}=\frac{\frac{C_{1}}{M T}}{\left(\begin{array}{c}
2 C_{o} v \\
M
\end{array}\right)\left(s+\frac{1}{T}\right)\left(s+\frac{1}{\tau}\right)}
$$

The above system described by the third order system with its TF having time delay approximation [XVI]. Without linearize the model the calculations become tough. So, the TF is linearized. The advantage of linearization of the model reduces the complexity of the problem calculations [XVIII].

\section{CONTROLLER DESIGN}

Let us define the plant model that will be used for MPC control [XVII]. First, we get a discrete-time version of the linearized model: 


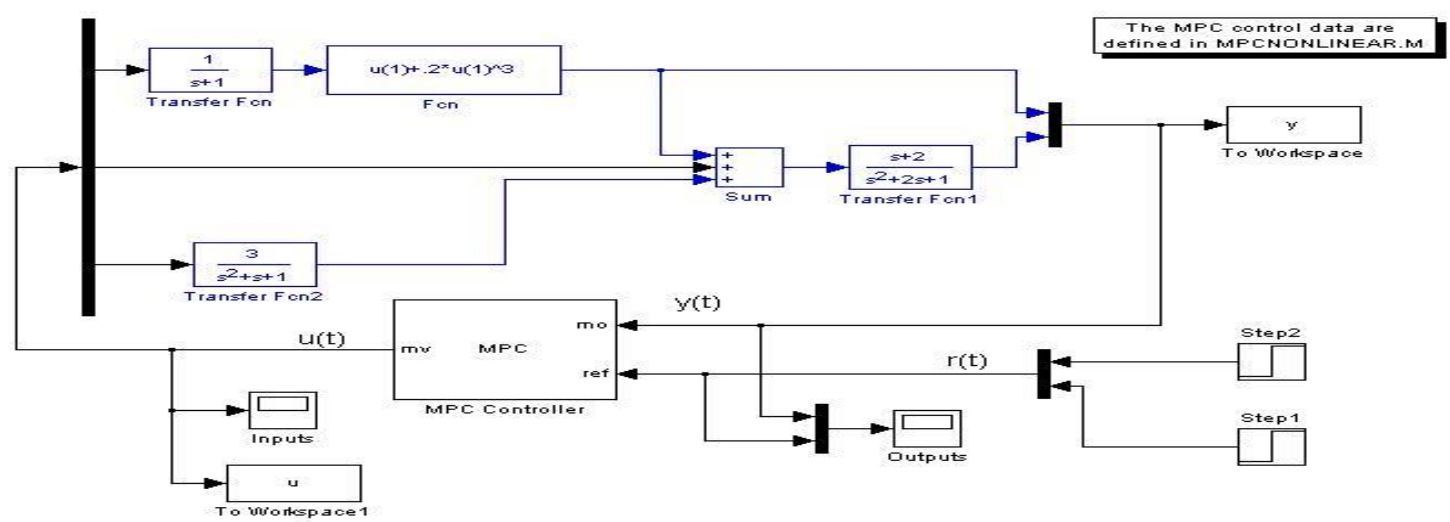

Figure (iii): MPC control of a MIMO nonlinear system

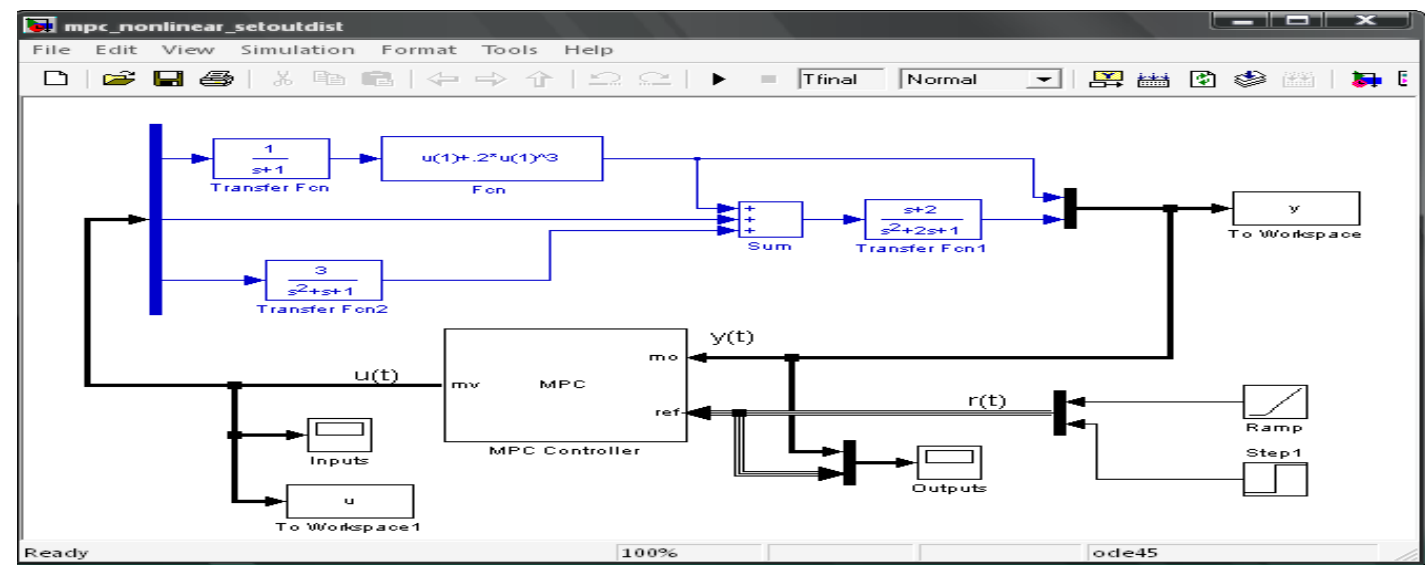

Figure (iv): Using an output disturbance model to track a ramp signal

\section{SIMULATION RESULTS}
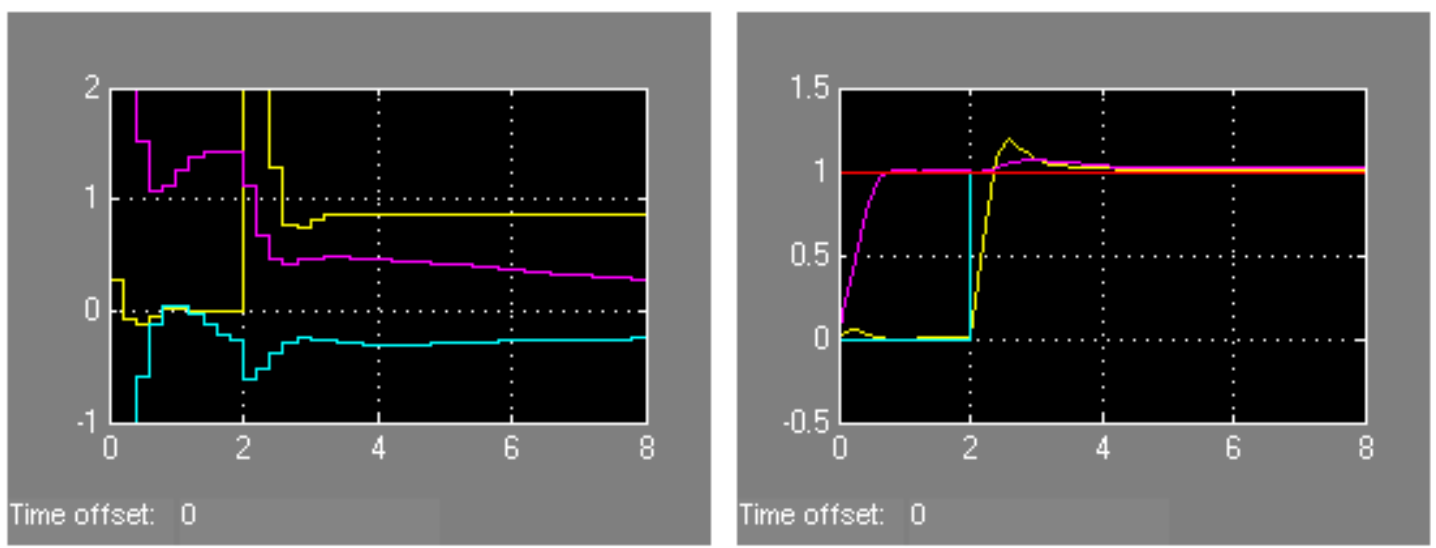

Figure (v): MPC control of a MIMO nonlinear system 


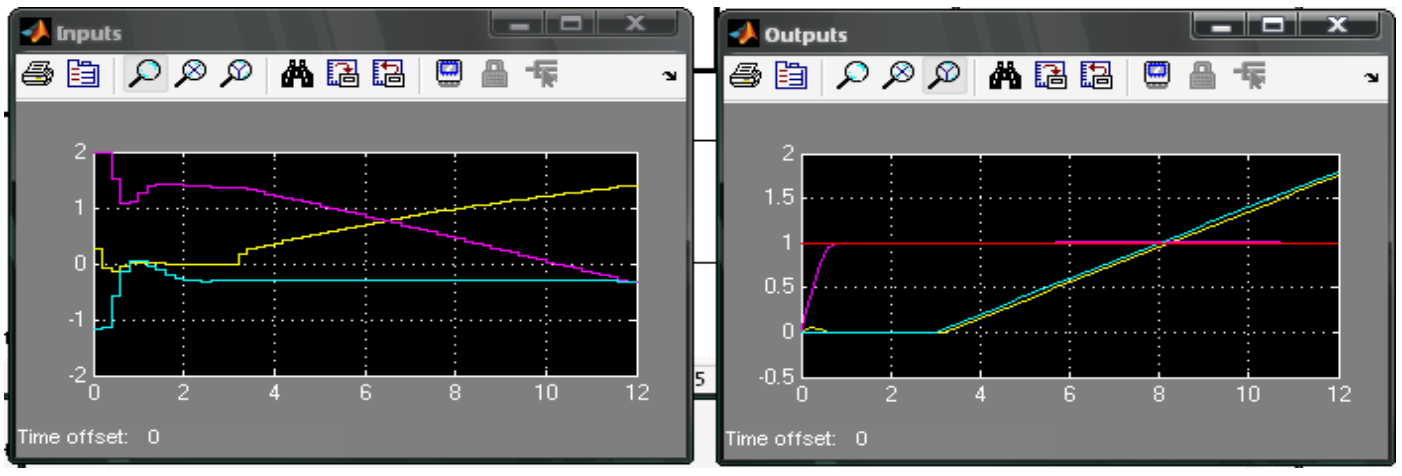

Figure (vi): Output disturbance model to track a ramp signal

\section{CONCLUSIONS}

The simulink mathematical model for cruise control system has been derived successfully for linear and nonlinear systems. With the implementation of conventional, modern and fuzzy controllers designed to control the cruise control system. The simulation results prove that the feed forward PID controller nullify the gravitational and wind disturbance effect in nonlinear systems. It is observed that one suitable controller is used to attain the desired response or output. We studied the analysis of nonlinear system by implementing the PID controller.

\section{REFERENCES}

1. . Datta, G. J. Silva, and S. P. Bhattacharyya, "New Results on the Synthesis of PID Controllers, " IEEE Transactions on Automatic Control, Vol. 47, No. 2, 241-252, February 2002.

2. Datta, H. Xu, and S. P. Bhattacharyya, “Computation of All Stabilizing PID Gains for Digital Control Systems," IEEE Transactions on Automatic Control, Vol. AC-46, No. 4647-652, April 2001.

3. Datta, M. T. Ho and S. P. Bhattacharyya, Structure and Synthesis of PID Controllers, Springer-Verlag, 2000.

4. F.Lin and W.M.Wonham, "On observability of discrete event systems, "Inf. Sci., vol. 44,pp. 173-198, 1988

5. F. Lin, S. Lafortune, and W. Wang "An algorithm for calculating indistinguishable states and clusters in finite-state automata with partially observable transitions," Syst. Control Lett., 2007.

6. Control System Design by Graham C. Goodwinl Stefan F.

7. J. Åström,Astrom K and T. Hägglund, PID Controllers: Theory, Design, and Tuning, Instrument Society of America, North Carolina, 1995.

8. J.E.Hopcroft and J.D.Ullman, Introduction to Automata Theory, Languages and Computation. Reading, MA; Addison-Wesley, 1979.

9. K.Rohloff, T.-S. Yoo, and S. Lafortune, “Deciding co-observability is PSPACE-complete,” IEEE Trans. Autom. Control, vol. 48, no. 11, pp.1995-1999, Nov. 2003.

10. K.Rudie, S.Lafortune, and F.Lin, "Minimal communication in a distributed discrete-event system, "IEEE Trans. Autom. Control, vol. 48,no. 6, pp. 957-975, Jun. 2003.

11. K.Rudie, S. Lafortune, and F. Lin, "Minimal communication in a distributed discrete-event control system," in Proc. Amer. Control Conf.,San Diego, CA, Jun. 1999, pp. 1965-1970.

12. K.Rudie and W.M.Wonham, “Think globally, act locally: Decentralized supervisory control,” IEEE Trans. Autom. Control, vol. 37, no. 11,pp. 1692-1708, Nov. 1992. 
13. L.H.Keel, J.I.Rego and S.P.Bhattacharyya, "A New Approach to Digital PID Controller Design" IEEE Trans. Aut. Contr. Vol. AC-48(4), pp.687-692, April 2003.

14. M.Sampath, R.Sengupta, S.Lafortune, K.Sinnamohideen, and Teneketzis, "Diagnosability of discrete event systems," IEEE Trans. Autom. Control, vol. 40, no. 9, pp.1555-1575, Sep. 1995.

15. Model Control techonolgy\& components by Kilian.

16. Modern Control Engineering by OGATA Fifth Edition PHI Publisher.

17. Power system engineering by Kothari \&Nagarath,Second Edition,Tata McGRAW HILL.

18. P.J.G.Ramadge and W. M. Wonham, "The control of discrete event systems," Proc.IEEE, vol. 77, no. 1, pp. 81-98, Jan. 1989.

19. Roland S. Burns Professor of Control Engineering Department of Mechanical and Marine Engineering University of Plymouth, UK by Advanced Control Engineering. 

